F. L.J. Plumb, Ueber regenerierten Kautschuk. (The India Rubber Journ. vom 11. Juli 1914.)

Enthält eine Besprechung der verschiedenen Methoden der Kautschukregeneration.

André Helbronner und Gustave Bernstein, Ueber die Vulkanisation von Kautschuklösungen mittels ultravioletter Strahlen. (C. r. d.l'Acad. des sciences 158, $1343-45$.)

Bei der Behandlung von schwefelhaltigen Kautschuklösungen mit ultravioletten Strahlen bildet sich ein sehr beständiges Gel. In diesem kann auch nach mehreren Monaten erst durch 18stündiges Erhitzen auf $80^{\circ}$ Fällung bewirkt werden. Nach Verdunsten des Lösungsmittels bleibt ein vulkanisiertes Häutchen zurück. Bei der Bestrahlung der Kautschuklösung mit ultraviolettem Licht entsteht aus dem gelösten Schwefel kolloider Schwefel, welcher sich je nach dem Maße seiner Bildung im Entstehungs- zustand mit dem Kautschuk verbindet. Setzt man den Schwefel in äußerst feiner Verteilung der ursprünglichen Kautschuklösung zu, dann erhält man im ultravioletten Licht keine Vulkanisation. Der Schwefelgehalt des vulkanisierten Häutchens ist viel geringer $(0,6$ Proz.) als der des vulkanisierten Kautschuks im allgemeinen. Die vulkanisierende Wirkung dieser geringen Schwefelmenge schließt nach $\mathrm{Hel}$ bronner und Bernstein eine katalysatorische Wirkung von Spuren von Schwefel ähnlich wie bei der Kautschuksynthese aus Isopren in sich.

Rudolf Ditmar, Die Vulkanisation von Guttapercha-Harz. (Gummi-Ztg. 28, Nr. 41, 1626.)

Guttapercha-Harze nehmen bei der Vulkanisationstemperatur Schwefel auf. Zum Nachweise dessen wurde eine Matschappij-Guttapercha, deren Harzgehalt 8,55 Proz. betrug, mit verschiedenen Mengen Schwefel auf Ebonit vulkanisiert. Die Untersuchung ergab folgende Zahlen :

\begin{tabular}{|c|c|c|c|c|c|c|}
\hline Nr. & $\begin{array}{l}\text { Analytisch be- } \\
\text { stimmt. Gesamt- } \\
\text { schwefel in der } \\
\text { Mischung } \\
\text { Proz. }\end{array}$ & $\begin{array}{l}\text { Gesant - Azeton- } \\
\text { Extrakt in der } \\
\text { Mischung } \\
\text { Proz. }\end{array}$ & $\begin{array}{l}\text { Freier Schwefel } \\
\text { i. Azetonextrakt, } \\
\text { berechnet auf die } \\
\text { Mischung } \\
\text { Proz. }\end{array}$ & $\begin{array}{c}\text { Gesamt-Azeton- } \\
\text { Extrakt auf die } \\
\text { Guttapercha be- } \\
\text { rechnet } \\
\text { Proz. }\end{array}$ & $\begin{array}{c}\text { Harzgehalt der } \\
\text { Guttapercha nach } \\
\text { Abzug des freien } \\
\text { Schwefels } \\
\text { Proz. }\end{array}$ & $\begin{array}{l}\text { Menge des rul- } \\
\text { kanisierten Har- } \\
\text { zes anf die Gutta- } \\
\text { percha berechnet } \\
\text { Proz. }\end{array}$ \\
\hline $\begin{array}{l}1 \\
2 \\
3 \\
4\end{array}$ & $\begin{array}{l}20,83 \\
25,74 \\
28,50 \\
31,90\end{array}$ & $\begin{array}{l}6,14 \\
4,61 \\
4,41 \\
7,12\end{array}$ & $\begin{array}{l}0,76 \\
1,02 \\
1,01 \\
2,64\end{array}$ & $\begin{array}{l}7,68 \\
6,01 \\
5,96 \\
9,97\end{array}$ & $\begin{array}{l}6,74 \\
4,16 \\
4,58 \\
6,28\end{array}$ & $\begin{array}{l}1,81 \\
4,39 \\
3,97 \\
2,27\end{array}$ \\
\hline
\end{tabular}

Bis zu zirka 26 Proz. Schwefelzusatz zur Guttapercha steigt auch die Menge des vulkanisierten Harzes. Ueber diesen Schwefelzusatz hinaus fällt merkwürdigerweise die vulkanisierte Menge des Harzes wieder ab. Es macht also den Eindruck, als ob sich das vulkanisierte Harz in einem Ueberschuß von Schwefel wieder auflöst, und daßj diese erhaltene Lösung von vulkanisiertem Harz in Schwefel azetonlöslich ist.

\title{
Ueber das allgemeine Verhalten der Elemente Schwefel, Selen und Tellur in Natron-Kalziumsilikatgläsern, mit besonderer Berücksich- tigung der durch dieselben bewirkten Färbungen.
}

\author{
Von Piero Fenaroli (Mailand). ${ }^{1}$ ) (Eingegangen am 7. Januar 1915.)
}

Die Analogie, welche zwischen den Elementen Schwefel, Selen und Tellur bezüglich ihrer Fähigkeit Färbungen zu erzeugen besteht, (Wien).

1) Aus dem Italienischen iibersetzt von J. Matula und die in Hinsicht auf die "Ultramarine" bereits von J. H of $\mathrm{mann}^{2}$ ) hervorgehoben wurde, ist in der Folge von P. Fenaroli bestätigt worden, der eingehend das Verhalten des 2) J. Hoffmann, Chem. Centr. 1906, [II] 720. 
Selens $^{3}$ ) und Tellurs ${ }^{4}$ ) in Natron-Kalziumsilikatgläsern untersuchte. Hierbei beobachtet man gleichfalls einen auffälligen Parallelismus zwischen dem in diesen Gläsern mit jenen Elementen stattfindenden Färbungsmechanismus und dem Färbungsmechanismus der Ultramarine im Sinne Hoffmanns. Demnach sind sowohl der Schwefel als das Selen und Tellur nur dann imstande, in den erwähnten Gläsern Färbungen hervorzurufen, wenn sie im Elementarzustand oder aber in Form von Polysulfiden bezw. Polyseleniden oder Polytelluriden der im Glas anwesenden Metalle, also höchstwahrscheinlich der Alkalimetalle, anwesend sind.

Betrachtet man eingehender letztere, durch die Polysulfide etc. bewirkte Färbungsform, so bemerkt man sofort die nahe Verwandtschaft zur ersteren durch die freien Elemente bewirkten, insbesonders, wenn man die Frage mehr von einem physikalischen, als vom rein chemischen Gesichtspunkte untersucht. Tatsächlich haben die Polysulfide, Polyselenide und Polytelluride $z$. B. der Alkalimetalle im allgemeinen Eigenschaften, die nicht merklich von jenen einer Schwefel- bezw. Selen- oder Tellurlösung in dem entsprechenden Monosulfid bezw. -Selenid oder-Tellurid abweichen. Und in der Tat, wie oft wurden in der Literatur Polyderivate dieser Elemente beschrieben, die sich bei genauerer Untersuchung als Lösungen eines Uebersciusses des Metalloides in dem entsprechenden metallischen Monosalze oder in einem Polyderivat niedrigeren Grades erwiesen! Die Konstitutionsformeln dieser Polyderivate, die neuestens namentlich für die Polysulfide angegeben und erörtert wurden, zeigen nur ein Atom des Metalloides direkt in Verbindung mit dem Metall, während die anderen in verschiedener Weise aber nur direkt untereinander verkettet sind ${ }^{5}$ ).

Dies vorausgeschickt, können wir nun alle möglichen, in Natron-Kalkgläsern durch jene drei Elemente bewirkten Färbungen ohne Zwang als eigentlich diese Elemente selbst ansehen, die überhaupt nur von der ver-

3) Chem.-Ztg. 1912, 1149, und 1914, 177.

4) Chem.-Ztg. 1914, 873 .

5) Die ausgeführten Untersuchungen beziehen sich in erster Linie auf den Schwefel. Vgl. W. Spring und J. Dim arte a u, Bull. soc. chim. 3, 1, 311, und namentlich F.W. Küster u. E. Heberlein, Zeitschr. f. anorg. Chem. 43, 53, Holm b e rg, Chem. Centr. 1908, [I] 1611 und 1910, [I] 734, H. Erd m an n, Chem. Centr. 1908, [II] 1089, J. A m a n n, Chem. Centr. 1911, [I] 1676 und $A$. Rule und J.S. Thom as, Chem. Centr. 1914, [I] 1058 . schiedenen Art und Weise ihrer Lösung oder genauer gesprochen, von ihrem verschiedenen Dispersionsgrade in der Lösung abhängen. Diese Auffassung ist auch in bester Uebereinstimmung mit der Konstitution des Glases selbst, das man mit E. Zschim mer ${ }^{6}$ ) als „den Inbegriff einer unendlich variablen Gruppe von amorph erstarrten Schmelzprodukten mineralisch saurer Oxyde in salzartiger Mischung mit basischen Oxyden" ansehen kann.

Während also die chemischen Eigenschaften des färbenden gelösten Elementes und die physikalischen Zustände des lösenden Glases über die Möglichkeit und die verschiedenen Erscheinungsformen der Färbung entscheiden, steht letztere selbst bei jedem der drei Elemente Schwefel, Selen und Tellur nur mit dem Dispersionsgrad in Beziehung, in welchem sich das disperse, färbende Element befindet, wenigstens solange dieses nicht infolge besonderer Umstände seinen eigenen Zustand ändert. Auf diese Weise erklärt sich vollständig die vollkommene Analogie der durch diese Elemente bewirkten Färbungen und der vollkommene Parallelismus ihrer Aenderungen in Gläsern und in anderen Lösungsmitteln.

Mit Schwefel gefärbte Gläser. Wie bekannt, erhält man mit Schwefel, solange die Gläser keine Schwermetalle enthalten, je nach der Konzentration nur gelbe oder gelbbraune Färbungen; die erhaltenen Gläser sind optisch leer. In diesen Gläsern finden sich Sulfide mit überschüssigem Schwefel, also wahrscheinlich Polysulfide vor; tatsächlich weisen diese Gläser eine Farbe auf, die gleich jener der wässerigen Lösungen von Alkalipolysulfiden ist, die ja gleichfalls hochdispersen Schwefel enthalten und daher ähnlich gefärbt erscheinen wie Schwefeldämpfe. Der stark elektronegative Charakter des Schwefels gestattet, falls er in einem Alkali-Kalkglas von gewöhnlicher $\mathrm{Zu}$ sammensetzung gelöst ist, bei den reduzierenden Zuständen während des Schmelzens kein Auftreten in anderer Form, als der einer echten Lösung in Anwesenheit von Sulfiden (Polysulfiden), und demnach auch kein Zustandekommen anderer Färbungen. In einem BorNatronglas hingegen, das stark sauren Charakter besitzt, ist die Möglichkeit eines Auftretens des Schwefels im freien Elementarzustand größer, demnach kann dieses auch blau gefärbt sein. (Hoff mann l. c.)

6) Zeitschr. f. Elektrochem. 11, 629, Chem. Centr. 1905, [II] 1200. 
Mit Selen gefärbte Gläser. Dieses Element hat einen mehr elektropositiven Charakter als der Schwefel und demnach ist die Möglichkeit, auch bei Anwesenheit der in den Natron-Kalkgläsern enthaltenen Alkalien im Elementarzustande aufzutreten, größer und es kann daher eine größere Mannigfaltigkeit der Färbung auftreten. Man kennt hier außer den verschiedenen Uebergangsfarben und einer einem speziellen Umformungszustand entsprechenden Färbung zwei Hauptfärbungen ${ }^{7}$ ). Bei sehr alkalireichen Gläsern und stark reduzierenden Bedingungen beobachtet man während dem Schmelzen kastanienbraune Färbungen, identisch mit jenen der Selendämpfe und der wässerigen Lösungen der Alkalipolyselenide. Diese Gläser enthalten Selenide und Selen im Ueberschuß, also wahrscheinlich Polyselenide; sie sind optisch leer, d. h. sie enthalten Selen im Zustande einer echten Lösung ${ }^{8}$ ); sie weisen also eine Färbung auf, die in Hinsicht auf den Bildungsmechanismus vollständig analog ist der durch Schwefel bedingten Färbung.

In nicht übermäßig alkalischen Gläsern von einer Basizität, die z. B. gleich oder nur etwas stärker ist als jene des "Normalglases", und bei nicht zu stark reduzierender Wirkung der Schmelzbedingungen kann das Selen vollständig dissoziieren oder sich wenigstens zum größten Teile von den Alkalimetallen im Elementarzustand freimachen und so zur Bildung kolloider Lösungen Anlaß geben. Dies sind die roten oder rosenfarbenen Gläser, die man gewöhnlich in der Industrie, nach den jetzt schon alten Patenten von Welz, Spitzer u. a. erzeugt.

Interessant ist, daß die in diesen Gläsern vorhandenen Submikronen des Selens sehr klein und einander sehr genähert sind. Diese Gläser zeigen auch bei der ultramikroskopischen Untersuchung einen Lichtkegel von derselben Farbe, die sie im durchfallenden Lichte aufweisen und der sich nur mittelst mikroskopischer Objektive von sehr hohem Auflösungsvermögen in unterschiedliche Submikronen auflösen läßt. In dem von P. Fenaroli ${ }^{9}$ ) eingehend untersuchten Falle gelang die Auflösung in deutliche Submikronen noch nicht mit der Wasserimmersion $D^{*}$ von $Z$ e i $B$, sondern erst mit der gleichfalls Zeiß'schen Homogenimmersion 1/12". Nun ist bekannt, daß die kleinste mittels eines Mikroskopobjektives wahrnehmbare Entfernung

7) P. Fenaroli, Chem.-Ztg. 1912, 1149, und $1914,177$.

8) P. Fenaroli, 1. c.

9) P. Fenaroli, Chem.-Ztg. 1914, 177. $\frac{\lambda}{a}$ beträgt, wobei $\lambda$ die Wellenlänge des der Farbe des Untersuchungsobjektes entsprechenden Lichtes und a die numerische Apertur des Objektives bedeuten. Da nun a für das Objektiv $D^{*}$ den Wert 0,75 , für das Objektiv $1 / 12^{\prime \prime}$ den Wert 1,30 hat und die Wellenlänge des der blauen Farbe der Submikronen entsprechenden Lichtes ungefähr $0,48 \mu$ beträgt, so kann man schließen, daß die mittlere Entfernung jener Submikronen etwa zwischen 0,64 und $0,37 \mu$ liegt. Mit Hilfe dieser Grenzwerte kann man annähernd auch die Dimensionen der Submikronen nach der Zsigmondy'schen Formel $1=\nu \sqrt[3]{\frac{\mathrm{A}}{\mathrm{S}}}$ berechnen, wobei 1 die Seitenlänge der als würfelförmig betrachteten Submikronen, $v$ ihre gegenseitige Entfernung, A die Menge der dispersen Substanz z. B. in $\mathrm{mg}$ pro $\mathrm{cmm}$ und $\mathrm{s}$ das spezifische Gewicht derselben bedeuten. Im Falle der roten selenhaltigen Gläser ist es nicht leicht, den genauen Wert von A zu ermitteln, da die direkte analytische Bestimmung des im Glase anwesenden Selens vor allem wegen der starken Verdünnung desselben sehr schwierig ist; außerdem ist der Wert auch bei genauer Bestimmung nicht zuverlässig, da hiermit noch nicht gesagt ist, daB alles Selen im Elementarzustand anwesend und an der färbenden Wirkung beteiligt ist. Tatsächlich geht aus den Untersuchungen P. F e n aroli's hervor, daß diese Gläser wahrscheinlich Selenide und Selenite enthalten, die wie leicht verständlich keinen vorwaltenden Anteil an der Färbung nehmen, die aber, wenn man nach Zerstörung des Glases durch Flußsäure nur das elementare Selen isolieren will, untereinander reagieren und zum großen Teile oder gänzlich in elementares Selen übergehen ${ }^{10}$ ). $\mathrm{Daß}$ tatsächlich nur ein kleiner Teil des anwesenden Selens an dem Zustandekommen der eigentlichen Färbung beteiligt ist, wurde auch vollständig durch Versuche bestätigt, die im Laboratorium von $O$. N. W itt ${ }^{11}$ ) ausgeführt wurden. O. N. Witt meint auch diesbezüglich, daß bei der Bestimmung, wieviel Selen an der Färbung wirklich aktiv beteiligt ist, natürlich nur die kolorimetrische Methode zum Ziele führen kann; er hat auch schon mit der Ausführung sehr exakter Versuche begonnen, deren vorläufige Ergebnisse zum Teile schon

10) P. Fenaroli 1. c.

11) O. N. Witt, Sprechsaal 1914, 444-445. 
publiziert wurden ${ }^{12}$ ). Die endgültigen Resultate werden auch in Hinsicht darauf, daß die Färbungsintensität einer kolloiden Lösung einerseits eine Funktion der Konzentration ist, anderseits aber auch in enger Beziehung zum Dispersitätsgrad der dispersen Substanz steht, interessant sein. Die Lösungen von kolloidem Selen mit bekanntem Titer in Gelatine, die Witt vorschlagen will und die zur Grundlage der kolorimetrischen Messung dienen sollen, müssen, falls sie zuverlässige Schlußfolgerungen gestatten sollen, das Selen in dem nämlichen Dispersionsgrad enthalten, in welchem es in den Gläsern vorkommt; dieser ist aber direkt oder indirekt die unbekannte Größe, die man in den Gläsern zu bestimmen sucht. Wit that bisher noch nicht angedeutet, wie er diese Frage lösen will.

Mir erscheint der erste und vielleicht auch der einzige Weg der Lösung dieser Frage folgender zu sein: eine Vergleichslösung von bekanntem Tit herzustellen, in welcher die einzelnen Submikronen im Ultramikroskope ein mittleres Reflexionsvermögen für Licht, d. h. eine photometrisch gemessene Helligkeit haben, die bei identischen Beleuchtungsbedingungen gleich jenem der entsprechenden Submikronen des Glases ist. Submikronen, die auf diese Weise übereinstimmen, müßten unter der Bedingung, daß das Lösungsmittel in beiden Fällen ungefärbt oder gleich gefärbt ist, die gleichen Dimensionen aufweisen. Die roten Selengläser enthalten aber nach den auch durch die Versuche von Fr. Kraz $\mathrm{e}^{13}$ ) bestätigten Beobachtungen Fenaroli's wechselnde Mengen von Seleniden, die sicherlich an der Nuance der Färbung nicht unbeteiligt sind und sicherlich die Durchsichtigkeit und das Lichtabsorptionsvermögen des Glases, in welchem sich die Submikronen des Selens verteilt vorfinden, beträchtlich beeinflussen werden. Es scheint daher sehr schwierig zu sein, auch auf kolorimetrischem Wege zur exakten Bestimmung des Wertes von A kommen zu können.

P. F.enaroli (1. c.) beschränkte sich daher darauf, einen maximalen Grenzwert der Größe der Submikronen des Selens in den von ihm untersuchten Gläsern zu bestimmen, wobei er, was sicherlich übertrieben ist, annahm, daß alles im Glase befindliche Selen nach dem Schmelzen im Elementarzustande daselbst vorhanden ist und als solches eine färbende Wirkung im Glase hervorruft. Der Gehalt des zugesetzten

12) Sprechsaal 1.c.

13) Ft. Kraze, Sprechsaal 1912, 214.
Selens war bekannt und betrug $0,00183 \mathrm{mg}$ pro cmm Glas und das spezifische Gewicht des Selens ist 4,5. Führt man in die oben angegebene Formel den maximalen Wert für $\nu$ $(0,64 \mu)$ ein, so ergibt sich, daß die Seitenlänge der als würfelförmig betrachteten Submikronen sicher kleiner sein muß als $47 \mu$, oder aber wenn man, was logischer ist, für $v$ den Mittelwert aus den beiden gefundenen Grenzwerten für $\nu$ (beispielsweise $0,50 \mu$ ) einsetzt, kleiner sein muß als $37 \mu \mu$. Alles in allem kann man sagen, daß die Submikronen des in dem in Rede stehenden Glase enthaltenen Selens, falls man sie als Würfel betrachtet, eine Seitenlänge haben, die im Mittel sicherlich kleiner als $40 \mu \mu$ ist; sie sind also sicherlich viel kleiner als jene von J. Reissigit) in den von ihm untersuchten Selenhydrosolen gemessenen.

Die Farbe der rosenfarbenen Selengläser variiert, wie aus der Praxis bekannt ist und durch die Untersuchungen P. Fenaroli's und die aus dem Laboratorium O. N. Witt's stammenden Arbeiten bestätigt wurde, beträchtlich mit dem Anstieg der Basizität; und zwar geht der Farbton ins gelblichbraune über. Dies ist offenbar die Folge einer Mehrbildung von Seleniden und Polyseleniden, wodurch der Dispersionsgrad des elementaren Selens zunimmt und die Färbung sich immermehr jenem braunen Ton der oben erwähnten optisch leeren Gläser nähert.

Die nämlichen rosenfarbenen Gläser erleiden bei sehr langsamer Abkühlung ${ }^{15}$ ) eine tiefgreifende Veränderung in dem Sinne, daß ihre Färbung mehr braun und opaleszent wird. Dies läßt sich unter der Annahme des folgendermaßen zusammengesetzten Gleichgewichtssystemes befriedigend erklären:

$3 \mathrm{Na}_{2} \mathrm{O}+(2 \mathrm{n}+1) \mathrm{Se} \leftrightarrows 2 \mathrm{Na}_{2} \mathrm{Se}_{n}+\mathrm{Na}_{2} \mathrm{SeO}_{3}$ welches System die Tendenz zeigt, sich bei hoher Temperatur nach links, bei relativ niedriger Temperatur nach rechts $z u$ verschieben.

Derartigen Gleichgewichtssystemen, obgleich bisher in Gläsern noch wenig untersucht, begegnet man sehr häufig in glasartigen Massen; dafür ist uns vor allem ein Beispiel in der Bildungsart des Avanturinglases nach V. Au g e ${ }^{16}$ ) gegeben; andere Beispiele stellen vielleicht einige kürzlich von A. Granger untersuchte kupferhaltige Gläser vor ${ }^{17}$ ). Es ist sehr wahr-

14) J. Re issig, Ann. d. Phys. 27, [4] 195 (1908).

15) P. Fenaroli l. c.

16) V. Auger, Compt. rend. 144, 422 (1907).

17) A. Granger, Bull. soc. chim. 15, 115 (1914). 
scheinlich, daß eine vernünftige und immer größere Verbreitung der modernen physikalischchemischen Ideen der glastechnischen und keramischen Chemie, sowohl in wissenschaftlicher als auch in praktischer Hinsicht sehr nützlich und wertvoll sein dürfte.

Andere wichtige, in der Praxis bekannte und im Laboratorium bestätigte Farbvariationen weisen bei Gleichheit aller übrigen Bedingungen die mit Selen gefärbten Natrongläser gegenüber den entsprechenden Kaliumgläsern auf; letztere haben ein viel reineres Rot, während erstere einen gelbbraunen Stich zeigen. Obgleich es nicht ausgeschlossen ist, daß hier auch chemische Umstände eine Rolle spielen, dürfte die Ursache hiervon doch wahrscheinlich physikalischer Natur sein. Wie schon P.Fenaroli (1. c.) andeutete, sind die Kaliumgläser bei gleichen sonstigen Bedingungen bekannterweise viel zäher als die entsprechenden Natrongläser, und infolge der gröBeren Zähigkeit des Mediums ist das bei hoher Temperatur dissoziierte elementare Selen weniger der Umwandlung in Selenide unterworfen, die bei der Abkühlung, während die Masse noch flüssig ist, erfolgt. Die Kaliumgläser werden demnach jene sein, deren Submikronen größer sind, wodurch sie eine rötere Farbe aufweisen (was wir noch besser in der Folge sehen werden), welche Tatsache vielleicht manchen im ersten Momente als im Widerspruche mit dem gewöhnlichen Verhalten der Kolloide erscheinen wird.

Einer anderen wichtigen Farbenänderung sind die Selengläser beim Erwärmen unterworfen. Nach den im Laboratorium von O. N. Witt (l. c.) gemachten Beobachtungen werden diese Gläser beim Erhitzen immer lichter, bis schließlich bei $620^{\circ}$ die Färbung völlig verschwindet.

Ich konnte diese Beobachtung überhaupt bestätigen, aber bei Untersuchung von Gläsern von einer Dicke von $2-3 \mathrm{~cm}$ konnte ich feststellen, daß die Farbe nicht verschwindet, sondern sie immer mehr in ein blasses Bräunlichgelb übergeht, das viel weniger intensiv als das Rot ist; bei über $600^{\circ}$ ist die ganze ursprüngliche Färbung in diesen Farbton umgewandelt. Wenn man bedenkt, daß das Selen sich gerade zwischen 600 und $700^{\circ}$ in Dampfform verwandelt, so versteht man leicht, daß es entweder wegen dieser Zustaindsänderung oder einfach wegen der erhöhten Dispersion oder aber aus beiden Gründen zu jener Farbwandlung kommt.

Das Selen ruft also in Gläsern, je nach dem Dispersionsgrad, in dem es sich in diesen vorfindet, verschiedene Färbungen hervor; dies bewog Fenaroli dazu, diese Gläser mit anderen Lösungen in ähnlichen Lösungsmitteln und besonders mit den "Bor-Selen-Uitramarinen" J. Hoffmann's ${ }^{18}$ ) zu vergleichen. Es wurden so selenhaltige Bor-Natrongläser hergestellt, die, stark erhitzt und hernach rasch abgekühlt, kolloides Selen enthielten und gelb gefärbt waren. Hielt man sie aber eine Zeitlang bei einer entsprechend niedrigen Temperatur im Schmelzzustande, so wurden die Gläser immer röter und schließlich sogar trüb, wobei sich erwies, daß sie Submikronen von Selen von immer wachsenden Dimensionen enthielten. Auch hier wie in den Natron-Kalkgläsern zeigt sich die Tendenz einer Farbenwandlung vom gelben ins rote, wenn das hochdisperse Selen einen niedrigeren Dispersitätsgrad annimmt. Dieses Verhalten ist in vollkommener Uebereinstimmung mit jenem des Selens in anderen Lösungsmitteln und befolgt genau das Ostwald'sche Gesetz, welches besagt, daß mit der Zunahme des Dispersionsgrades sich das Absorptionsmaximum im Spektrum gegen die kürzeren Wellen zu verschiebt.

$\mathrm{Da}$ die Lichtabsorption des Selens im $\mathrm{Zu}$ stand höchster Dispersion in einer vollkommenen Absorption des ganzen sichtbaren Spektrums vom Grün bis zum Violett besteht, während die größere Submikronen enthaltenden, eigentlichen kolloiden Selenlösungen (und nicht die groben Suspensionen) nur noch eine Absorption aufweisen, die vom Gelbgrün durch das gesamte Violett geht, so folgt andererseits daraus, daß die verschiedenen kolloiden Selenlösungen nur Farben haben können, die vom Gelb zum Purpurrot oder Rotviolett gehen, wie dies auch vollständig von der Erfahrung bisher bestätigt wurde.

Mit Tellur gefärbte Gläse ${ }^{19}$ ). Das Tellur hat einen noch weniger ausgesprochenen elektronegativen Charakter als das Selen und kann demnach, wie auch die Erfahrung lehrt, noch leichter in Natron-Kalkgläsern im Elementarzustande abgeschieden werden und infolge Bildung kolloider Lösungen zur Entstehung verschiedener Färbungen Anlaß geben. Mit Tellur konnten korallen- und purpurrote Färbungen erhalten werden, die identisch jenen sind, welche die wässerigen konzentrierten Lösungen der Kaliumpolytelluride aufweisen. Derartige durch Tellur gefärbte Gläser erhält

18) J. H off m a n n, Chem. Centr. 1906 [II], 720 und 1912, [l] 382 .

19) P. Fenaroli, Chem.-Ztg. $1914,873$. 
man unter energischen Reduktionsbedingungen und bei Anwendung relativ großer Tellurmengen (zirka 0,15 Proz.)

Die Analyse ergab, daß jene Gläser Telluride, bei Ueberschuß von Tellur also wahrscheinlich Polytelluride enthalten und daß dieselben nicht optisch leer sind. Die in diesen Gläsern anwesende disperse Phase hat einen gröBeren Dispersitätsgrad als in den andern noch zu erwähnenden mit Tellur gefärbten Gläsern von brauner oder blauer Farbe. Aus den Veränderungen der Konzentration der in diesen roten Gläsern enthaltenen Submikronen und ihrer Beziehung zur Intensität der Färbung konnte jedoch nicht festgestellt werden, dab diese Submikronen tatsächlich das Farbpigment bilden; es ist nicht unwahrscheinlich, daß es sich um eine kolloiddisperse Substanz handelt, die keinen direkten Einfluß auf die Färbung hat. Der im Ultramikroskope zu beobachtende Lichtkegel von blasser blauvioletter Farbe ist mit dem Ze i B'schen Objektiv $D^{*}$ schwer auflösbar. Die Auflösung erfolgt hingegen vollständig mit Hilfe der Zeiß'schen Homogenimmersion $1 / 12$ "; alle sichtbaren Submikronen sind von gleichem Aussehen. Das heißt aber, daß, wenn diese kolloid-disperse Substanz, wie es den Anschein hat, nicht das Pigment vorstellt, dieses letztere sich in einem noch höheren Dispersionsgrad, vielleicht sogar im molekular-dispersen Zustand befinden muf. Das Absorptionsspektrum derartig gefärbter Gläser ist identisch mit jenem der wässerigen Lösung des Kaliumpolytellurids und umfaßt wie dieses eine allgemeine $\mathrm{Ab}$ sorption des gesamten Teiles des Spektrums zwischen Orange bis einschlieblich zum Violett mit einem charakteristischen Maximum bei ungefähr $480-490 \mu \mu$.

Neben diesen Gläsern kann man mit Tellur (immer unter reduzierenden Schmelzbedingungen) bei bloßer Aenderung der Konzentration, ganz analog wie bei den Hydrosolen, verschieden gefärbte kolloide Lösungen erhalten, und zwar bei niedriger Konzentration des Tellurs eine braune, bei höherer eine stahlblaue; letztere Färbung ist durch Submikronen von größeren Dimensionen als jene, die die Braunfärbung bestimmen, bedingt.

Versuche, bleihaltige Gläser und Glasuren mit Tellur zu färben, waren — wie übrigens vorauszusehen war - vollständig negativ, da man ohne energische Reduktion nur durchsichtige farblose Gläser erhält, während es bei stattfindender Reduktion infolge der Entstehung von Bleitellurid und der Zersetzung des Bleiglases zur Bildung schwärzlicher Massen kommt.

Mit Zunahme des Dispersitätsgrades des Tellurs im Glase beobachtet man also analog dem Verhalten der Hydrosole, sogar noch deutlicher, einen Uebergang der Färbung von Blau zu Braun und Rot.

Dies ist in vollster Uebereinstimmung mit. dem Ostwald'schen Gesetz sowie eine schöne Bestätigung desselben, nach welchem „mit zunehmendem Dispersionsgrad der dispersen Phase das Absorptionsmaximum im Spektrum sich nach den kürzeren Wellen zu verschiebt“.

Mailand, den 4. Januar 1915.

Aus dem Privatlaboratorium
des Verfassers.

\title{
Die Einwirkung von Elektrolyten auf organische Abwässer.
}

\author{
Von Paul Rohland (Stuttgart). (Eingegangen am 5. Jaunar 1915.)
}

Kolloid veranlagte Silikate, natürliche wie Ton, Kaolin' ${ }^{1}$ ), Talk und künstliche wie Zement, Ultramarin usw. können durch Zusatz von bestimmten Elektrolyten zur raschen Sedimentation gebracht werden, z. B. Kaolinsuspensionen durch Zusatz von Hydroxylio ne $n$ in bestimmter Konzentration, Ultramarinund Zementsuspensionen durch Zusatz von verdünnten Lösungen von $\mathrm{Kalzi}$ m chlorid, Kalziumsulfat und Natriumchlorid.

1) Vg1. P. Rohl a nd, Die Einwirkung von Hydro$x$ vionen auf Kaolinsuspensionen. (Koll.-Zeitschr. 1912.)
Nur auf kolloid veranlagte Stoffe findet diese Einwirkung statt, weil sie in Berührung mit Wasser kolloide Substanzen, die Hydroxyde des Siliziums, Aluminiums, Magnesiums und Eisens bilden.

Berliner Blau ist kolloider Natur und gehört auch hierher.

Dagegen werden alle $\mathrm{kr}$ istalloide $\mathrm{n} \mathrm{Sub-}$ stanzen, gleichviel ob organischer oder anorganischer Natur, z. B. Bariumsulfat durch Zusatz von Elektrolyten nicht sedimentiert.

Es ist merkwürdig, daß di e Elektrolyte, die am stärksten auf die Sedimentation mancher 